\title{
Biotinidase Deficiency
}

National Cancer Institute

\section{Source}

National Cancer Institute. Biotinidase Deficiency. NCI Thesaurus. Code C84598.

A genetic disorder caused by mutations in the BTD gene. It is characterized by reduced or absent activity of the enzyme biotinidase which is responsible for the recycling of the vitamin biotin. Signs and symptoms appear in childhood and include seizures, hypotonia and developmental delays. If left untreated, it leads to vision and hearing loss, infections, alopecia and ataxia. 